\title{
Accelerated launch of video visits in ambulatory neurology during COVID-19
}

Key lessons from the Stanford experience

\author{
Laurice Yang, MD, MHA, Cati G. Brown-Johnson, PhD, Rebecca Miller-Kuhlmann, MD, \\ Samantha M.R. Kling, PhD, RDN, Erika A. Saliba-Gustafsson, PhD, Jonathan G. Shaw, MD, MS, \\ Carl A. Gold, MD, MS, and Marcy Winget, PhD
}

Neurology ${ }^{\circledR}$ 2020;95:305-311. doi:10.1212/WNL.0000000000010015

\section{Abstract}

The SARS-CoV-2 (COVID-19) pandemic has rapidly moved telemedicine from discretionary to necessary. Here, we describe how the Stanford Neurology Department (1) rapidly adapted to the COVID-19 pandemic, resulting in over 1,000 video visits within 4 weeks, and (2) accelerated an existing quality improvement plan of a tiered roll out of video visits for ambulatory neurology to a full-scale roll out. Key issues we encountered and addressed were related to equipment/software, provider engagement, workflow/triage, and training. On reflection, the key drivers of our success were provider engagement and dedicated support from a physician champion, who plays a critical role understanding stakeholder needs. Before COVID-19, physician interest in telemedicine was mixed. However, in response to county and state stay-athome orders related to COVID-19, physician engagement changed completely; all providers wanted to convert a majority of visits to video visits as quickly as possible. Rapid deployment of neurology video visits across all its subspecialties is feasible. Our experience and lessons learned can facilitate broader utilization, acceptance, and normalization of video visits for neurology patients in the present as well as the much anticipated postpandemic era.

\author{
Correspondence \\ Dr. Yang \\ layang@stanford.edu
}

\section{MORE ONLINE}

COVID-19 Resources

For the latest articles, invited commentaries, and blogs from physicians around the world

NPub.org/COVID19

From the Department of Neurology \& Neurological Sciences (L.Y., R.M.-K., C.A.G.), Stanford University School of Medicine; and Evaluation Sciences Unit (C.G.B.-J., S.M.R.K., E.A.S.-G., J.G.S., M.W.), Stanford University School of MedicineCA.

Go to Neurology.org/N for full disclosures. Funding information and disclosures deemed relevant by the authors, if any, are provided at the end of the article. 


\section{Glossary}

APP = advanced practice provider; COVID-19 = SARS-CoV-2; EMR = electronic medical record; SHC $=$ Stanford Health Care.

Although telemedicine supported by video on patients' personal electronic devices has been in use for several years, it has had relatively limited and focused use in neurology, with sparse evaluation of its feasibility across neurology's numerous ambulatory subspecialties. ${ }^{1,2}$ Telemedicine's ability to provide access to rural or smaller hospitals without subspecialists is a recognized advantage. ${ }^{3,4}$ However, challenges including technical and connectivity issues, ${ }^{5}$ limitations with the physical examination, ${ }^{6-11}$ and lack of personal interaction are nontrivial issues that must be addressed to enable broad implementation of neurologic video visits. ${ }^{10}$ These and other challenges ${ }^{1,9,11-16}$ have resulted in variable and incremental uptake within the ambulatory neurology setting. With the onset of the SARSCoV-2 (COVID-19) pandemic, the applicability of and necessity for video visits changed and was rapidly put to the test across all subspecialties in neurology. ${ }^{17-19}$

Here, we describe Stanford Neurology Department's transition of all subspecialty and general neurology clinics to video visits. We demonstrate feasibility and share early learnings as we (1) rapidly adapted to the COVID-19 pandemic, resulting in over 1,000 video visits within 4 weeks, and (2) accelerated an existing quality improvement plan of tiered roll out of video visits for ambulatory neurology to a full-scale deployment for the entire department. We discuss key issues and associated solutions. Our experience and lessons learned can be useful to other health care organizations identifying and implementing strategies to meet patient care needs while containing infection risk with COVID-19 and practicing stay-at-home orders.

\section{Context}

\section{Institutional context}

Within the United States, California was a leader in achieving relatively broad SARS-CoV-2 testing and thus identified a rapid increase in new COVID-19 cases. As a result, the San Francisco Bay Area, including Santa Clara County, was one of the first regions in the United States to implement stay-athome advisories (March 16, 2020) followed by a Californiawide order on March 19. These orders, combined with the desire to protect patients and health care workers from infection, resulted in Stanford Health Care (SHC), announcing that all clinics must have the capability to convert all patient visits to video visits by April, 2020.

\section{Structure of Stanford Neurology ambulatory clinics}

The Stanford Neurology Department comprises 12 subspecialties with approximately 60 faculty and 8 advanced practice providers (APPs: advanced practice providers). Trainees include approximately 36 residents and 28 fellows. The neurology clinics are spread over 4 floors of 2 buildings: 3 clinical examination floors and 1 floor for procedures.

\section{Adapting the original quality improvement plan to the pandemic}

The initial quality improvement plan was to rollout video visits across providers and clinics within 4 subspecialties relatively slowly, and deliberately, using a step-wedge design with two 5-week testing phases and pseudo-randomization of physicians and APPs to the first or second cohort. The purposeful rollout was intended to optimize workflow and identify changes needed to improve and ensure acceptability and value for patients, neurologists, and APPs before expanding to all $\sim 70$ providers (both neurologists and APPs) over the next several months. The original plan comprised a scheduled soft-launch date of March 16, 2020, to include 2 providers and 1 scheduler followed by a full launch of video visits in the first cohort the following week, March 23. In addition, we had originally elected to use the video functionality built into our electronic medical record (EMR) software, which required visits to be conducted on official clinic computers. In light of the pandemic, we had to quickly adapt our plan to enable a rapid broad launch of video visits across all providers and subspecialties.

\section{Institutional review board}

We obtained institutional review board exemption for quality improvement from Stanford (protocol \#55644).

\section{Key adaptations and factors for successful rapid launch of video visits}

The soft-launch date was converted to a full launch on March 16,2020 , to rapidly enable video visits for all physicians and APPs in neurology. In-clinic computers were available for use during the week of March 16, and all SHC-issued computer laptops and desktops were enabled by March 23, 2020. The issues we surmounted to enable the rapid and broad launch fall into 4 categories: (1) equipment: hardware and software set-up and troubleshooting; (2) physician engagement; (3) process workflow development for (re)scheduling and triaging patients; and (4) training for medical assistants, schedulers, and providers.

\section{Equipment: hardware-challenges and how we addressed them}

Equipment we needed for the widespread launch included approximately 50 clinic desktops and 50 home computer laptops and desktops (to allow providers to work primarily from home 
due to stay-at-home orders and disruption of childcare services). About 15 were already enabled for video visits as part of our original launch plan, but we had to urgently obtain more. All devices and software were encrypted and Health Insurance Portability And Accountability Act (HIPPA) compliant. Although providers had asked to use their phones or tablets, these devices were unfortunately not compatible with the current system, Epic Hyperspace (Verona, Wisconsin); the software required specific installation and activation by the SHC information technology department to enable video visit capabilities as well as a microphone and speaker sound check. This required a close partnership with our local SHC information technology department to rapidly deploy the necessary hardware and software with appropriate approvals and was not feasible to do on providers' personal devices.

In our original, prepandemic, quality improvement plan, physicians and APPs were not going to be able to conduct video visits from home due to the cost of the necessary laptops. However, when faced with sudden inability to safely provide the majority of our typical outpatient care, the cost/ benefit analysis clearly shifted, and resources were mobilized and approved rapidly at the hospital administrative level.

We had also initially anticipated the need for specialized headsets for optimal audio input and output during the visits. We found, however, that the specialized headsets were not necessary; existing computer internal audio/microphone worked without significant detriment to the experience.

\section{Equipment: software-challenges and how we addressed them}

We initially chose to activate the video functionality within the medical record system at our institution through Epic Hyperspace. This software allows patients to log on through the Stanford Healthcare MyHealth patient-facing interface using an app on their smartphone, tablet, or a home computer. The physician initially needed to log on from an official SHCencrypted clinic computer either in the clinic space or an SHC-issued computer approved for home usage. Subsequent iterations of video visits have enabled physicians to log on to Epic Hyperspace from personal computers that have been encrypted by SHC IT.

Several challenges occurred with the video interface during visits. Because the technical issues were not routinely reported or documented, it was unclear which type of problem was the most common or whether the problems were mostly from the patient or provider side. Based on ad hoc communications, however, one of the most common issues seemed to be malfunctioning of the video or audio. When this occurred, it was usually addressed by conversion of the video visit to a phone visit. Although this workaround was feasible, it was not an ideal solution in the clinic setting as rooms were not always equipped with phones. A chat function embedded in the video software had been considered as a potential way to establish real-time tech support, but was not currently available.
Another issue was that multiprovider conferencing was not available with the originally chosen platform (although it will be available soon); this created significant challenges to our medical training program. It was not possible for providers to see patients with trainees unless the trainee and the attending were in the same room, thus violating pandemic social distancing restrictions. In response, we developed and rolled out a parallel structure within 10 days of the initial stay-at-home order using the HIPAA-compliant Zoom (San Jose, California) platform in our clinics that involved any trainees (i.e., residents, fellows, and medical students). We rapidly developed a second set of instructions for the process flow for video visits using Zoom including trainees, precepting attendings, schedulers, and patients. We were able to teach residents and schedulers the new process within 2 days once it was developed. This was implemented by March 26, 2020, for all the clinics' residents, fellows, and medical students.

Based on the equipment and software challenges that were identified quickly, several changes to functionality will be made, including a new software platform that will allow multiprovider conferencing through Epic Hyperspace (rendering Zoom unnecessary), screen-share capability, and ability to use a non-hospital-issued personal laptop that is Stanford compliant, specifically, encrypted for HIPAA and Protected Health Information use.

\section{Physician engagement}

We began the process of engaging physicians and APPs on video visits in January 2020 for our original prepandemic quality improvement project. We were greeted at that time with mixed interest, ranging from excitement to try a new technology and increase clinic flexibility and accessibility to not interested at that time. Before COVID-19, the subset of physicians who were interested and curious about video visits had agreed to do 2-3 visits per week. After the stay-at-home order in response to the COVID-19 pandemic, all physicians converted the majority of their patients to video visits. The dramatic change in circumstances surrounding COVID-19 led to a corresponding dramatic and immediate increase in physician and APP engagement. Thus, this unusual situation became an unexpected catalyst for change, resulting in universal training, fluency, and comfort with a new technology that we anticipate would have otherwise taken years to adopt.

\section{Workflow/triage process, challenges, and how we addressed them}

The workflow/triage process for identifying scheduled visits that would be appropriate for video visits and subsequent (re) scheduling is shown in figure, A. Figure, B shows the workflow process during a video consultation and how technical issues were addressed if they occurred. Briefly, beginning March 16, 2020 , providers identified patients who were clinically urgent and needed to retain the existing scheduled in-person visit and clinically nonurgent patients who could either have a video visit or defer their in-person visit to another date. Specific eligibility criteria are still under development, but the primary 

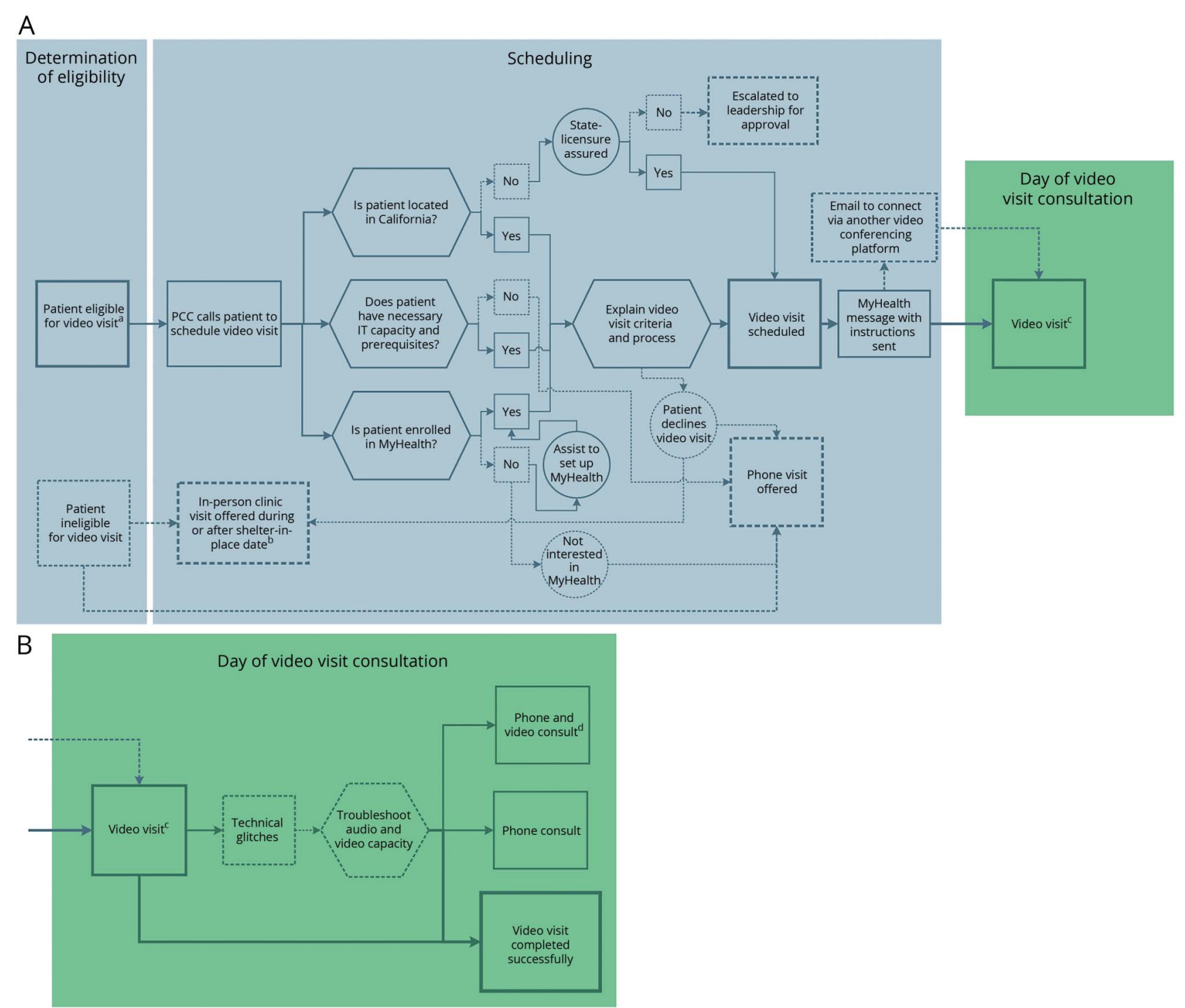

(A) Workflow process map-determination of patient eligibility, scheduling of video visit, and video visit consultation. (B) Workflow process map during video visit consultation. a Determined by physician/nurse/APP who assesses urgency and necessity of clinical examination. Determined by physician/nurse/

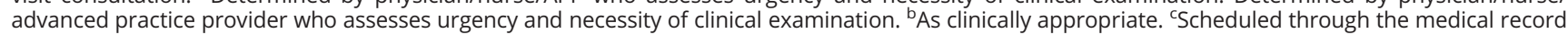
system at our institution or a different video conferencing platform that allows trainees and/or interpreters to be on the consultation when needed (during early implementation, the medical record system at our institution lacked multiple person functionalities). ${ }^{d}$ If a good audio connection is not retrievable, some providers use a combination of phone call and video call to consult with the patient, although troubleshooting was not centralized (this is an area in development). PCC = patient care coordinator.

reason for in-person visits was necessity of a hands-on physical examination.

The transition worked most seamlessly for follow-up patients with relatively stable or chronic complaints. Triaging new patient referrals, whether new to the department or to SHC, was more challenging. During this crisis, we continued supporting the workforce to process new patient referrals, and we continued to triage cases accordingly. This process required active engagement and review by providers; this was time consuming but necessary. For example, new patients with long-standing stable history of paresthesia referred for evaluation of neuropathy were generally deferred to a later inperson date, given the necessity of a hands-on examination. Patients with evolving symptoms, even if sensory only, were usually classified as urgent, so we either conducted a video visit to triage whether an in-person visit was indicated or immediately scheduled an in-person visit.

Ease of conversion to video visits varied across neurology subspecialty divisions because some subspecialties are more reliant on examinations that require in-person visits. For instance, patients with dementia do not necessarily need a strength examination, whereas with patients with amyotrophic lateral 
sclerosis or multiple sclerosis, several important aspects of the examination can be difficult to elicit via video. However, by March 30, 2020, providers had seen that initial video visits were useful and beneficial to patients, and therefore, the vast majority of patients were offered video visits without extensive case-bycase triage across all subspecialties. With this shift in mindset, we often used video visits more as a triage and developed a 2-stage visit, with initial video visit for the clinical history and online examination followed by a planned in-person visit for a detailed hands-on examination if needed. In-person consultations were thusly reserved for urgent procedures including botulinum injections, autonomic testing, EMG, and EEG.

After screening patients scheduled for the upcoming week, the provider notifies the scheduler. The scheduler is responsible for calling all patients identified as appropriate for a video visit to provide the options and explain (1) how to connect with the platform used by video visits; (2) benefits and risks of video visits in the context of COVID-19; (3) confirmation that patient resides in-state (due to licensure restrictions for telemedicine); and (4) assessment of patient IT capacity to perform video visits - patients need a smartphone or computer with camera capabilities. Stanford uses the SHC MyHealth app, which interfaces with the patient's EMR for notes, appointments, labs, imaging, and communication with care team. If the patient agrees to a video visit, they are scheduled for one; the scheduler then sends additional information to the patient via the secure patient portal regarding the process and regulations. If the patient refuses the video visit, usually due to inability to use the technology, they are offered the option of a phone call or to have their in-person visit rescheduled to a future date in approximately 1 to 2 months, depending on the patient's clinical status and the status of the stay-at-home order at the time of the interaction.

\section{Training and hands-on coaching}

Providers and schedulers were trained using a hospital-wide mandatory 15-minute online video module, which reviewed the EMR-based interface, safety basics such as checking patient identification, best practices for sound and lighting optimization, and building patient rapport (e.g., putting the video in the top center of the screen close to the computer camera to simulate eye contact). Following advice from other departments at Stanford with experience conducting video visits, the physician champion also conducted 1:1 training sessions on the first day of a provider's video visit, when feasible, reaching approximately $50 \%$ of providers. This additional 1:1 training provided many physicians with extra support they needed to get comfortable with video visits. This was a significant factor in successful deployment, as it helped providers quickly normalize their new work, making it easier to rapidly increase their video visit patient load.

Training for staff was available in different modalities to accommodate work schedules. During the stay-at-home orders, staff rotated whether they needed to be on campus and most worked from home. All trainings were $\sim 30$ minutes long and were conducted in person before social distancing policies or via WebEx or Zoom, which worked quite well as trainers leveraged screen share functions to troubleshoot issues as needed in early implementation. Schedulers also had additional training conducted virtually with the physician champion and/or clinic managers. The champion or manager provided additional resources to support the schedulers, including an email template to be sent to patients and process instructions in the form of a checklist to use when scheduling patients over the phone. After initial deployment of the new process flow, it was also very important to be available for questions or concerns to ensure that the workflow was implemented correctly. The physician champion joined virtual daily huddles, answered emails from staff in a timely manner, and frequently visited schedulers' workstations in person to answer questions.

\section{Training challenges and how we addressed them}

Because of the urgency of the COVID-19 pandemic and high provider engagement, we did not encounter significant training challenges with providers or staff. However, it was very important to continually reemphasize the vital role schedulers play when calling and educating patients how to use the video visit application. Emails to patients alone were insufficient to ensure patient understanding of the new technology. Therefore, these phone calls, though time consuming, were necessary to ensure successful video visit completion.

\section{Discussion}

The ambulatory clinic of the Stanford Neurology Department successfully rolled out video visits for most patients, across all subspecialty clinics, in just 3 weeks, with a dedicated clinic manager team, robust institutional support and a physician champion, who plays a critical role in balancing the staff/ physician interest and needs with clinical operations. Although we had a head start with the planning of the video visit implementation, which resulted in some of the hardware and software being in place and scheduling workflow developed, we had to urgently address additional and unexpected issues related to equipment, workflow, provider engagement, and training, as is the case with most technological roll-outs.

On reflection, we believe that provider engagement and a very supportive clinic manager team and physician champion were the key drivers for our success. The physician champion and clinical management team quickly addressed all logistical issues related to equipment and workflows. Indeed to implement change successfully, the important role of physician and nurse champions and teamwork has been highlighted for the uptake of telestroke. ${ }^{20-22}$ Champions facilitate, support, and troubleshoot administrative, IT, legal, and financial matters that arise when shifting toward telemedicine. ${ }^{21}$ They also play a crucial role in cultivating enthusiasm to ensure successful adoption of a telemedicine approach. 
In our department, physicians' mixed interest in video visits shifted dramatically post-COVID-19, when most patients who wanted to continue being seen were converted to video visits, including most new referrals and follow-ups previously scheduled. We are still investigating the impact of COVID-19 on our anticipated visit volumes and video visit completion rates in April and May 2020.

Early reactions and feedback from providers suggest promise for the long-term sustainability and utilization of video visits. This is based on early evaluation of 30-minute qualitative individual interviews that will eventually include approximately 30 providers and 10 staff. Providers found value in seeing their patients in their home and being able to engage with family members in a way not afforded by a phone call. In fact, it was robustly reported that video visits were way better than a phone call. This aligns with other studies that have noted the value of assessing patients in their own homes $6,23,24$ by, for example, helping identify safety issues, verifying patients' medications, ${ }^{25}$ and involving family members on the call. $^{26}$ Providers also expressed that there is huge potential for video visits, given the increased ability to monitor health data remotely, such as gait sensors and applications for commercially available smart devices to track movement. ${ }^{5,12,23,27}$ These positive responses to the value and potential of video visits indicate potential to maintain provider engagement as we transition out of the initial responses to the COVID-19 pandemic.

Many providers were pleased to be able to protect their most vulnerable patients from long-distance travel for in-person visits, which, even in times of good health, could set patients back in the course of their disease. The challenge with travel could be compounded by the fact that many neurology patients have serious physical and/or cognitive decline that make them dependent on others to get to and from clinics, making it very challenging to move the patient from their home environment. This could result in further barriers to appropriate and timely care. ${ }^{1,25}$ Video visits, however, have the potential for saving patients and/or their caregivers travel time and associated outof-pocket and health care costs. $1,5,8,23,25$

Providers also reported innovating on physical examination techniques. For example, Stanford neuro-ophthalmologists have demonstrated that performing an eye examination online is possible in their educational videos teaching others how to do eye examinations online (med.stanford.edu/ophthalmology/patient_care/tele-eyecare.html\#virtual_care).

Other studies have also found that many types of physical examinations, particularly those that are largely visual, can be effectively and safely performed via video. ${ }^{1,28,29}$ Through faceto-face virtual interactions, providers can assess patient movements by requesting the patient to perform or repeat tasks, although they may have limited ability to test for rigidity or assess the pull test adequately without someone assisting the patient on-site. ${ }^{23}$

\section{Conclusions and next steps}

Video visits were rapidly integrated into ambulatory neurology including multiple subspecialties, with over 1,000 video visits in 4 weeks. To do this, we surmounted issues related to computer equipment, physician engagement, workflow development, and training of providers and staff. Our next steps are to continue rigorous evaluation of rapid video visit implementation using mixed methods to assess both implementation $^{30}$ and effectiveness outcomes. We will assess both provider and patient perspectives of acceptability, appropriateness, and quality of care afforded by video visits. We aim to inform strategies to sustain neurology's expanded use of this modality and inform standards or guidelines for patients who are clinically appropriate for video visits.

If given the opportunity to launch video visits again, we would have worked to (1) build even more provider and patient buyin before implementation by sending out surveys and participating in meetings/support groups ahead of time so we could better investigate anticipated problems with the system and be prepared to address them up-front; (2) ensure timely device infrastructure before implementation; (3) make a single initial software solution available to address all use cases (singleprovider as well as multi-provider); and (4) better prepare physicians on how to do a neurologic exam online through a tele-neurology curriculum, which we are currently creating.

We hope reflections such as these, and descriptions laid out in this work overall will facilitate broader utilization, acceptance, and normalization of video visits into both the present as well as the much anticipated post-pandemic era.

\section{Acknowledgment}

The authors thank Dr. Frank Longo, Dr. Yuen So, Alison Kerr, and Sophia Loo for their support of clinical innovation. They thank Dr. David Larson and the leadership of the Improvement Capability Development Program for arranging the partnership between the Department of Neurology \& Neurological Sciences and the Evaluation Sciences Unit. They also thank Dr. Heather Moss, Dr. Shannon Beres, and the Stanford ophthalmology department for providing education materials on telemedicine neurology.

\section{Study funding}

This project was supported by Stanford Healthcare as part of the Improvement Capability Development Program.

\section{Disclosure}

The authors report no disclosures relevant to the manuscript. Go to Neurology.org/N for full disclosures.

\section{Publication history}

Received by Neurology April 20, 2020. Accepted in final form May 28, 2020. 
Appendix Authors

\begin{tabular}{|c|c|c|}
\hline Name & Location & Contribution \\
\hline $\begin{array}{l}\text { Laurice Yang, } \\
\text { MD, MHA }\end{array}$ & Stanford & $\begin{array}{l}\text { Implementation; design and concept; } \\
\text { drafted the manuscript; and significant } \\
\text { revisions }\end{array}$ \\
\hline $\begin{array}{l}\text { Cati Brown- } \\
\text { Johnson, PhD }\end{array}$ & Stanford & $\begin{array}{l}\text { Design and concept; data collection and } \\
\text { analysis; drafted the manuscript; and } \\
\text { significant revisions }\end{array}$ \\
\hline $\begin{array}{l}\text { Rebecca Miller- } \\
\text { Kuhlmann, MD }\end{array}$ & Stanford & $\begin{array}{l}\text { Implementation; design and concept; } \\
\text { drafted the manuscript; and significant } \\
\text { revisions }\end{array}$ \\
\hline $\begin{array}{l}\text { Samantha M.R. } \\
\text { Kling, PhD, RDN }\end{array}$ & Stanford & $\begin{array}{l}\text { Design and concept and drafted the } \\
\text { manuscript }\end{array}$ \\
\hline $\begin{array}{l}\text { Erika A. Saliba- } \\
\text { Gustafsson, PhD }\end{array}$ & Stanford & $\begin{array}{l}\text { Data collection and analysis and drafted } \\
\text { the manuscript }\end{array}$ \\
\hline $\begin{array}{l}\text { Jonathan G. } \\
\text { Shaw, MD }\end{array}$ & Stanford & $\begin{array}{l}\text { Design and concept; drafted the } \\
\text { manuscript; and significant revisions }\end{array}$ \\
\hline $\begin{array}{l}\text { Carl A. Gold, MD, } \\
\text { MS }\end{array}$ & Stanford & $\begin{array}{l}\text { Implementation; design and concept; } \\
\text { and significant revisions }\end{array}$ \\
\hline $\begin{array}{l}\text { Marcy Winget, } \\
\text { PhD }\end{array}$ & Stanford & $\begin{array}{l}\text { Design and concept; drafted the } \\
\text { manuscript; and significant revisions }\end{array}$ \\
\hline
\end{tabular}

\section{References}

1. Hatcher-Martin JM, Adams JL, Anderson ER, et al. Telemedicine in neurology: telemedicine work group of the American Academy of Neurology update. Neurology 2020;94:30-38.

2. Dorsey ER, Glidden AM, Holloway MR, Birbeck GL, Schwamm LH. Teleneurology and mobile technologies: the future of neurological care. Nat Rev Neurol 2018;14:285-297.

3. Davis LE, Harnar J, LaChey-Barbee LA, Richardson SP, Fraser A, King MK. Using teleneurology to deliver chronic neurologic care to rural veterans: analysis of the first 1,100 patient visits. Telemed J E Health 2019;25:274-278.

4. Demaerschalk BM, Berg J, Chong BW, et al. American Telemedicine Association: telestroke guidelines. Telemed J E Health 2017;23:376-389.

5. Schneider RB, Biglan KM. The promise of telemedicine for chronic neurological disorders: the example of Parkinson's disease. Lancet Neurol 2017;16:541-551.

6. Howard IM, Kaufman MS. Telehealth applications for outpatients with neuromuscular or musculoskeletal disorders. Muscle Nerve 2018;58:475-485.

7. Helleman J, Kruitwagen ET, van den Berg LH, Visser-Meily JMA, Beelen A. The current use of telehealth in ALS care and the barriers to and facilitators of implementation: a systematic review. Amyotroph Lateral Scler Frontotemporal Degener 2020;21:167-182.
8. Mammen JR, Elson MJ, Java JJ, et al. Patient and physician perceptions of virtual visits for Parkinson's disease: a qualitative study. Telemed J E Health 2018;24:255-267.

9. Hassan A, Dorsey ER, Goetz CG, et al. Telemedicine use for movement disorders: a global survey. Telemed J E Health 2018;24:979-992.

10. Geronimo A, Wright C, Morris A, Walsh S, Snyder B, Simmons Z. Incorporation of telehealth into a multidisciplinary ALS clinic: feasibility and acceptability. Amyotroph Lateral Scler Frontotemporal Degener 2017;18:555-561.

11. George BP, Scoglio NJ, Reminick JI, et al. Telemedicine in leading US neurology departments. Neurohospitalist 2012;2:123-128.

12. Achey M, Aldred JL, Aljehani N, et al. The past, present, and future of telemedicine for Parkinson's disease. Mov Disord 2014;29:871-883

13. Wechsler LR. Advantages and limitations of teleneurology. JAMA Neurol 2015;72: 349-354.

14. Reider-Demer MM, Eliashiv D. Expanding the use of telemedicine in neurology: a pilot study. J Mobile Technology Med 2018;7:46-50.

15. Dorsey ER, Topol EJ. State of telehealth. New Engl J Med 2016;375:154-161.

16. Patel UK, Malik P, DeMasi M, Lunagariya A, Jani VB. Multidisciplinary approach and outcomes of tele-neurology: a review. Cureus 2019;11:e4410.

17. Waldman G, Mayeux R, Claassen J, et al. Preparing a neurology department for SARS CoV-2 (COVID-19): early experiences at Columbia University Irving Medical Center and the New York Presbyterian Hospital in New York City. Neurology 2020;94: 886-891.

18. Guidon AC, Amato AA. COVID-19 and neuromuscular disorders. Neurology 2020; 94:959-969.

19. Klein BC, Busis NA. COVID-19 is catalyzing the adoption of teleneurology. Neurology 2020;94:903-904.

20. Dumitrascu OM, Demaerschalk BM. Telestroke. Curr Cardiol Rep 2017;19:85.

21. Wechsler LR, Demaerschalk BM, Schwamm LH, et al. Telemedicine quality and outcomes in stroke: a scientific statement for healthcare professionals from the American Heart Association/American Stroke Association. Stroke 2017;48:e3-e25.

22. Gibson J, Lightbody E, McLoughlin A, et al. "It was like he was in the room with us": patients' and carers' perspectives of telemedicine in acute stroke. Health Expect 2016; 19:98-111.

23. Ben-Pazi H, Browne $\mathrm{P}$, Chan $\mathrm{P}$, et al. The promise of telemedicine for movement disorders: an interdisciplinary approach. Curr Neurol Neurosci Rep 2018;18:26.

24. Dorsey ER, Vlaanderen FP, Engelen LJ, et al. Moving Parkinson care to the home. Mov Disord 2016;31:1258-1262.

25. Appireddy R, Khan S, Leaver C, et al. Home virtual visits for outpatient follow-up stroke care: cross-sectional study. J Med Internet Res 2019;21:e13734.

26. Morland LA, Poizner JM, Williams KE, Masino TT, Thorp SR. Home-based clinical video teleconferencing care: clinical considerations and future directions. Int Rev Psychiatry 2015;27:504-512.

27. Chirra M, Marsili L, Wattley L, et al. Telemedicine in neurological disorders: opportunities and challenges. Telemed JE Health 2019;25:541-550.

28. American Academy of Neurology. Telemedicine and COVID-19 implementation guide. In: Telemedicine and remote care [online].Available at: https://www.aan com/tools-and-resources/practicing-neurologists-administrators/telemedicine-andremote-care/. Accessed April 28, 2020.

29. Awadallah M, Janssen F, Korber B, Breuer L, Scibor M, Handschu R. Telemedicine in general neurology: interrater reliability of clinical neurological examination via audiovisual telemedicine. Eur Neurol 2018;80:289-294.

30. Proctor E, Silmere H, Raghavan R, et al. Outcomes for implementation research: conceptual distinctions, measurement challenges, and research agenda. Adm Pol Ment Health 2011;38:65-76.

\section{COVID-19 and Neurologic Disease: Call for Papers!}

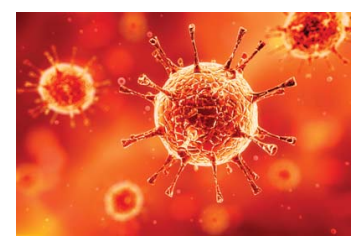

The editors of Neurology are interested in papers that address the neurological aspects of COVID-19 infection and challenges to the management of patients with chronic neurological conditions who have, or are at risk for, the infection. Relevant papers that pass initial internal review will undergo expedited peer review and online publication. We will consider papers posted in preprint servers.

Submit observational studies and clinical trials as Articles and case series and case reports under the Clinical/Scientific Notes category to https://submit.neurology.org/ today! 


\section{Neurology}

\section{Accelerated launch of video visits in ambulatory neurology during COVID-19: Key lessons from the Stanford experience}

Laurice Yang, Cati G. Brown-Johnson, Rebecca Miller-Kuhlmann, et al.

Neurology 2020;95;305-311 Published Online before print July 1, 2020

DOI 10.1212/WNL.0000000000010015

\section{This information is current as of July 1, 2020}

\section{Updated Information \&} Services

References

Citations

Subspecialty Collections

Permissions \& Licensing

Reprints including high resolution figures, can be found at: http://n.neurology.org/content/95/7/305.full

This article cites 29 articles, 5 of which you can access for free at: http://n.neurology.org/content/95/7/305.full\#ref-list-1

This article has been cited by 3 HighWire-hosted articles: http://n.neurology.org/content/95/7/305.full\#\#otherarticles

This article, along with others on similar topics, appears in the following collection(s):

All Clinical Neurology

http://n.neurology.org/cgi/collection/all_clinical_neurology

All Practice Management

http://n.neurology.org/cgi/collection/all_practice_management

Information about reproducing this article in parts (figures,tables) or in its entirety can be found online at:

http://www.neurology.org/about/about_the_journal\#permissions

Information about ordering reprints can be found online:

http://n.neurology.org/subscribers/advertise

Neurology ${ }^{\circledR}$ is the official journal of the American Academy of Neurology. Published continuously since 1951, it is now a weekly with 48 issues per year. Copyright () 2020 American Academy of Neurology. All rights reserved. Print ISSN: 0028-3878. Online ISSN: 1526-632X.

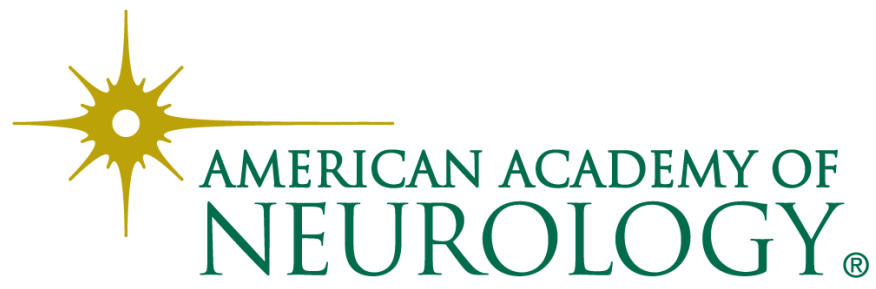

